OPEN ACCESS

Edited by:

Teresa Zotta,

Italian National Research Council

(CNR), Italy

Reviewed by:

Maria de los Angeles Serradell,

National Council for Scientific and Technical Research (CONICET),

Argentina

Alex Galanis,

Democritus University of Thrace,

Greece

${ }^{*}$ Correspondence:

Ruixia Gu

guruixia1963@163.com

${ }^{\dagger}$ These authors have contributed equally to this work

Specialty section:

This article was submitted to

Food Microbiology,

a section of the journa

Frontiers in Microbiology

Received: 06 July 2018 Accepted: 27 May 2019

Published: 12 June 2019

Citation:

Wa $Y$, Yin $B$, He $Y, X i W$, Huang $Y$, Wang C, Guo F and Gu R (2019)

Effects of Single Probioticand Combined Probiotic-Fermented Milk on Lipid Metabolism

in Hyperlipidemic Rats.

Front. Microbiol. 10:1312.

doi: 10.3389/fmicb.2019.01312

\section{Effects of Single Probiotic- and Combined Probiotic-Fermented Milk on Lipid Metabolism in Hyperlipidemic Rats}

\author{
Yunchao Wa ${ }^{1,2 t}$, Boxing Yin ${ }^{1,2 t}$, Yong $\mathrm{He}^{3}$, Wenbo $\mathrm{Xi}^{3}$, Yingping Huang ${ }^{3}$, Chunlei Wang ${ }^{3}$, \\ Feixiang $\mathrm{Guo}^{3}$ and Ruixia Gu${ }^{1,4 *}$
}

' Jiangsu Key Laboratory of Dairy Biotechnology and Safety Control, Yangzhou University, Yangzhou, China, ${ }^{2}$ College of Animal Science and Technology, Yangzhou University, Yangzhou, China, ${ }^{3}$ Uni-President China Holdings Ltd., Shanghai, China, ${ }^{4}$ College of Food Science and Engineering, Yangzhou University, Yangzhou, China

Previous studies have shown that probiotics have positive effects on hyperlipidemia by lowering the serum lipid concentration and improving the lipid profile. To explore the mechanism by which probiotic-fermented milk improves lipid metabolism, the transcription of genes regulated by liver $X$ receptors (LXRs), 5'-AMP-activated protein kinase, and the farnesoid $X$ receptor (FXR), which play integral roles in lipid metabolism, was investigated in hyperlipidemic rats. Compared with rats fed a high-fat diet, the administration of probiotic-fermented milk significantly lowered the levels of total cholesterol (TC) and total triglycerides (TG) in rat serum and viscera $(P<0.05)$ and significantly increased the level of total bile acid in the rat liver and small intestine $(P<0.05)$. The quantitative PCR results showed that the probiotics ameliorated the TC levels in the rats by activating the transcription of genes involved in the LXR axis, which promoted TC reverse transport and increased the conversion of TC to bile acids. The level of TG in the hyperlipidemic rats was ameliorated by the inhibition of the transcription of carbohydrate reaction element binding protein genes and activation of the transcription of PPAR $\alpha$ genes. The regulation of lipid metabolismrelated gene transcription by the single probiotic (Lactobacillus rhamnosus LV108)fermented milk was more effective than that by the combined probiotic (L. rhamnosus LV108, Lactobacillus casei grx12, and Lactobacillus fermentum grx08)-fermented milk $(P<0.05)$.

Keywords: lipid metabolism, hyperlipidemic rats, probiotics, fermented milk, LXRs, AMPK, FXR

\section{INTRODUCTION}

Hyperlipidemia is a common cardiovascular disease posing a serious threat to human health. With the change in dietary structure, high-fat diets have become an important factor in hyperlipidemia, causing the incidence of hyperlipidemia to increase each year. However, other cardiovascular diseases, such as atherosclerosis, coronary heart disease, and hypertension, are also potential risks. Mann (1974) observed the hypocholesterolemic activity of fermented milk in a Maasai tribe in Kenya. Subsequently, animal and human models have been used to evaluate the effects of probiotic 
microorganisms on serum lipid levels, and probiotic benefits have been emphasized over the last 40 years. Accumulating studies have shown that well-established probiotics, prebiotics, and synbiotics possess hypocholesterolemic and other effects that modulate serum lipids in humans and animals ( $\mathrm{He}$ and Shi, 2017). Currently, the mechanisms by which probiotics and their fermentation products reduce lipid levels mainly include the adhesion and absorption of living bacteria cells, the production of short chain fatty acids, the reduction of the reabsorption of bile acids, and the inhibition of lipoprotein lipase activity.

Recently, several studies have shown that liver $\mathrm{X}$ receptors (LXRs), 5'-AMP-activated protein kinase (AMPK), the farnesoid $\mathrm{X}$ receptor (FXR) and their downstream target genes play important roles in lipid metabolism (Tu et al., 2000; Geyeregger et al., 2006; Yao et al., 2016). LXRs, which are nuclear receptors activated by oxidized sterols, are widely expressed in the liver and small intestine and regulate some key genes in cholesterol metabolism (Zelcer and Tontonoz, 2006). The main function of LXRs includes limiting cholesterol accumulation in macrophages and other peripheral cell types by increasing the expression of the reverse cholesterol transporters ATP-binding cassette members A1 (ABCA1), G1 (ABCG1), and G5 (ABCG5), resulting in a program of reverse cholesterol transport (RCT) (Song et al., 1994; Willy et al., 1995; Tall et al., 2008). AMPK is a serine/threonine kinase widely found in eukaryotic cells that play an important role in maintaining the balance of cellular energy metabolism (Yao et al., 2016). Once activated, AMPK stimulates the activator of the oxide enzyme to activate receptor $\alpha(\operatorname{PPAR} \alpha)$, upregulating the related target genes, promoting fatty acid oxidation to enhance the body's catabolism, enhancing the $\beta$-oxidation of fatty acids, and promoting the production of ATP (Patel et al., 2014). AMPK can also inhibit the expression and transcriptional activity of sterol regulatory element binding protein (SREBP-1c) and carbohydrate reaction element binding protein (ChREBP), downregulating the transcription of related target genes and inhibiting lipid synthesis (Tu et al., 2000; Yoshikawa et al., 2001). Bile acids are endogenous FXR ligands expressed in the small intestine (Tu et al., 2000). FXRs can maintain the internal stability of bile acids by regulating the transcription of genes involved in bile acid metabolism (Tu et al., 2000). In addition, FXRs regulate the synthesis and transport of bile acids and prevent the excessive accumulation of bile acids in hepatocytes by inhibiting CYP7A1 transcription (Tu et al., 2000).

Recently, some studies have shown that probiotics can inhibit cholesterol uptake and increase cholesterol efflux by activating LXRs, eventually reducing the overall cholesterol level and potential risk of developing atherosclerosis (Huang et al., 2015). To the best of our knowledge, few studies have focused on the effects of probiotics on the multiple pathways of lipid metabolism or compared the effects of probiotics and combined probiotics on gene transcription in the lipid metabolism pathway. In this study, Lactobacillus rhamnosus LV108, Lactobacillus casei grx12, and Lactobacillus fermentum grx08 were isolated from human bodies at the Long Life Village in China (Chen, 2014). These three strains possess good acid resistance, artificial gastrointestinal tolerance, and bile salt tolerance in vitro. These three strains have been shown to improve the intestinal flora, antagonize pathogenic bacteria, modulate the immune response, possess anti-oxidation activity, and prevent tumors in animal models. Regarding blood lipid regulation, our previous study showed that L. rhamnosus LV108, L. casei grx12, and L. fermentum grx08 can reduce cholesterol in vivo and in vitro (Chen, 2014; Huang et al., 2013; Liu et al., 2016).

In this study, hyperlipidemic rats were used as a model to investigate the effects of fermented milk containing L. rhamnosus LV108, L. casei grx12, and L. fermentum grx08 on the transcription levels of lipid metabolism genes. Simultaneously, the effects of single probiotic-fermented milk and a mixture of milks fermented with three probiotics on hyperlipidemic rats were analyzed and compared.

\section{MATERIALS AND METHODS}

\section{Probiotics and Preparation of Milk Fermented With Probiotics}

The starter cultures (L. rhamnosus LV108, L. casei grx12, and L. fermentum grx08) were provided by Jiangsu Province Key Laboratory of Dairy Processing and Safety, Yangzhou University. Each activated culture was inoculated into de Man, Rogosa, and Sharpe (MRS) medium (Haibo Biotechnology Co., Ltd., Qingdao, China) and incubated at $37^{\circ} \mathrm{C}$ for $12 \mathrm{~h}$.

Skim milk $(12 \%, \mathrm{w} / \mathrm{w})$ was heat-treated at $95^{\circ} \mathrm{C}$ for $15 \mathrm{~min}$ and cooled to $37^{\circ} \mathrm{C}$. The milk was fermented with L. rhamnosus LV108, L. casei grx12, or L. fermentum grx08 at $37^{\circ} \mathrm{C}$ for $20 \mathrm{~h}$ and stored at $4^{\circ} \mathrm{C}$. The L. rhamnosus LV108-fermented milk was fermented with L. rhamnosus LV108, and the combined probiotic-fermented milk was a mixture of the three milks fermented with a single probiotic strain at a $1: 1: 1(w: w: w)$ ratio. The number of viable bacteria in each milk fermented with a single probiotic strain and the combined probiotic-fermented milk was quantified to ensure that the number of viable bacteria was above $1 \times 10^{10} \mathrm{cfu} \cdot \mathrm{mL}^{-1}$. The two probiotic-fermented milks used in this study contained the same total viable count.

\section{Animals, Diet, and Experimental Design}

Forty male Sprague-Dawley rats aged 5 weeks and weighing $140 \pm 4.5 \mathrm{~g}$ were purchased from the Comparing Medical Center of Yang Zhou University (Jiangsu, China). The rats were individually housed in stainless steel cages in a pathogen-free room at a controlled temperature $\left(22 \pm 2{ }^{\circ} \mathrm{C}\right)$ with a relative humidity of $55 \pm 5 \%$ under a $12 \mathrm{~h}$ light-dark cycle. All animal procedures complied with the Guide for the Care and Use of Laboratory Animals and were approved by the Animal Care Committee of the Centers for Disease Control and Prevention (Jiangsu Province, China).

After 1 week of acclimation, the rats were randomly divided into four groups with 10 individuals per group. The groups included a control group (C), a high-fat model group (HF), a single probiotic (L. rhamnosus LV108)-fermented milk group (HFPB), and a combined probiotic-fermented milk (the mixture consisted of L. rhamnosus LV108-fermented milk, L. casei grx12-fermented milk, and L. fermentum grx08-fermented milk) group (HFPBS). Before the experiment, the rats in all groups, 
except for those in the $\mathrm{C}$ group, were fed a high-fat diet for 4 weeks, and their blood was collected to determine whether they exhibited hyperlipidemia. During the experiment, the rats in all groups, except for those in the $\mathrm{C}$ group, continued to be fed a high-fat diet.

All treatments were implemented by means of intragastric administration. The administered dose was determined according to the weight of the rats $\left(1 \mathrm{~mL} 100 \mathrm{~g}^{-1}\right)$, and continuous intragastric administration was performed for 4 weeks. The $\mathrm{C}$ and $\mathrm{HF}$ groups were treated with normal saline, the HFPB group was treated with milk fermented with L. rhamnosus LV108 (total viable count $>1 \times 10^{10} \mathrm{cfumL}^{-1}$ ), and the HFPBS group was treated with combined probioticfermented milk containing a mixture of milks fermented with L. rhamnosus LV108, L. casei grx12, and L. fermentum grx08 (total viable count $>1 \times 10^{10} \mathrm{cfu} \cdot \mathrm{mL}^{-1}$ ). The animal groups and treatments are shown in Table 1.

\section{Measurement of Physiological and Biochemical Indexes Weight Measurement in the Rats}

The rats were weighed regularly each week, and the changes in weight were recorded. The dose administered via the gavage feeding was adjusted according to the body weight of the rats.

\section{Measurement of the Rat Viscera Index}

After 4 weeks of gavage feeding, the rats were weighed. The spleen, liver, and heart were removed, and the surrounding tissues were cleaned and weighed.

\section{Serum and Viscera Biochemical Analysis}

The total triglycerides (TGs), total cholesterol (TC), total bile acids (TBAs), high-density lipoprotein cholesterol (HDL-C), and low-density lipoprotein cholesterol (LDL-C) were determined using a Hitachi BS-7020 Automated Chemistry Analyzer (Hitachi Limited, Japan) using the corresponding TG, TC, TBA, HDL$\mathrm{C}$, and LDL-C kits purchased from the Nanjing Jiancheng Bioengineering Institute (Nanjing, Jiangsu, China). The very low-density lipoprotein cholesterol (VLDL-C) was determined using ELISA kits purchased from Shanghai Hualan Chemical Technology Co., Ltd. (Shanghai, China).

\section{Histopathological Examination}

The liver was removed, rinsed with physiological saline solution, blotted dry with filter paper, and weighed. The pathological lesions were observed, and the relative liver weights were calculated. The liver samples were immersion fixed, stored in $4 \%$ poly-formaldehyde solution, and routinely processed by embedding in paraffin. Five-micrometer sections were cut and stained with hematoxylin and eosin ( $\mathrm{H} \& \mathrm{E})$. The preparation of the tissue for the histopathology examination was conducted according to the methods described by Zhang et al. (2012).

\section{Real-Time Quantitative PCR (qPCR) Analysis of the Transcription of Genes}

The total RNA from the liver and small intestine was isolated using an UNlQ-10 Column TRIzol Total RNA Isolation Kit
TABLE 1 | Animal groups and treatments.

\begin{tabular}{lll}
\hline Group & Diet & Gavage \\
\hline Control (C) & Normal diet & Normal saline \\
High fat (HF) & High-fat diet & Normal saline \\
High fat with probiotics & High-fat diet & L. rhamnosus LV108 \\
(HFPB) & & Fermented milk \\
High fat with probiotics & High-fat diet & Mixture consisting of milk \\
(HFPBS) & & fermented with \\
& & L. rhamnosus LV108, \\
& & L. casei grx12, and \\
& & L. fermentum grx08 \\
\hline
\end{tabular}

(Sangon Biotech Co., Ltd., Shanghai, China) according to the manufacturer's protocol. The tissue samples from each single group were pooled for the RNA extraction. The primers used for the reference gene ( $\beta$-actin) and target genes were designed using the Primer 5.0 online software and are shown in Table 2. Quantitative real-time PCR was performed using an ABI Prism 7300 Detection System (Applied Biosystems, United States). All reactions were performed in duplicate using a kit provided by TaKaRa Biotechnology Company.

The relative gene mRNA levels were determined using the $\Delta$ cycle threshold ( $\Delta \mathrm{Ct})$ method, and $\beta$-actin served as a reference gene. For each target gene, the $\Delta \Delta \mathrm{Ct}$ values of all samples were calculated by subtracting the average $\Delta \mathrm{Ct}$ of the $\mathrm{C}$ group from the average $\triangle \mathrm{Ct}$ of the HF, HFPB, or HFPBS group. The $\Delta \Delta \mathrm{Ct}$ values were converted to fold differences by raising 2 to the power of $-\Delta \Delta \mathrm{Ct}$ (i.e., $2^{-\Delta \Delta \mathrm{CT}}$ ).

\section{Statistical Analysis}

All results are expressed as the mean \pm standard deviation (SD). The differences among the groups were determined using a one-way ANOVA, and significant differences were considered significant at $P<0.05$. SPSS software version 21.0 was used for all analyses.

\section{RESULTS}

\section{Body Weight and Organ Indexes}

The weights of the rats and organs after dissection were determined to calculate the organ indexes. The final weights of the HF, HFPB, and HFPBS groups were significantly higher than the final weight of group $C(P<0.05$, Table 3$)$. The maximum increase in weight after 4 weeks was $225.34 \mathrm{~g}$ and occurred in the HF group, while the minimum value was $208.05 \mathrm{~g}$ and occurred in the HFPB group. The liver index values in the HF, HFPB, and HFPBS groups were significantly higher than those in the $C$ group $(P<0.05$, Table 3$)$, but there were no significant differences in the other organ indexes.

\section{Lipid Profiles}

The physiological and biochemical indexes in the serum, liver, and small intestine of the rats were quantified. In the serum, the TC, TG, VLDL-C, LDL-C, and TBA values in the HF group were significantly higher than those in the $C$ group 
TABLE 2 | List of genes measured in liver and small intestine tissues isolated from rats.

\begin{tabular}{|c|c|}
\hline & Primer sequences $\left(5^{\prime}-3^{\prime}\right)$ \\
\hline $\operatorname{LXR} \alpha(\mathrm{F})$ & CAGAGCCTACAGAACTTCGT \\
\hline $\operatorname{LXR} \alpha(\mathrm{R})$ & CTCGCAGCTCAGCACATT \\
\hline $\operatorname{LXR} \beta(F)$ & AAGCTGGTGAGCCTGCGC \\
\hline $\operatorname{LXR} \beta(R)$ & CGGCAGCTTCTTGTCCTG \\
\hline $\mathrm{ABCA1}(\mathrm{F})$ & GGTGGTGTTCTTCCTCGTTAC \\
\hline $\mathrm{ABCA1}(\mathrm{R})$ & GCTTCCGCTTCCTTCTGTAG \\
\hline ABCG1(F) & GCCTGGCCATTGCACTAGAAC \\
\hline$A B C G 1(R)$ & GGACACCACTTGGAAGCAAGA \\
\hline ABCG5(F) & CGCAGGAACCGCATTGTAA \\
\hline ABCG5(R) & TGTCGAAGTGGTGGAAGAGCT \\
\hline SREBP-1C(F) & GCTGATGGAGACAGGGAGTT \\
\hline SREBP-1C(R) & GCAGTTGATGTAGAGGCTAAGC \\
\hline ChREBP(F) & CGAGGTGGTGATGCGTGAAT \\
\hline ChREBP(R) & GAAGTTTGAAGATGTGGGCGT \\
\hline $\operatorname{AMPK}(\mathrm{F})$ & CAGGCACATGGTTGTCCACAG \\
\hline $\operatorname{AMPK}(\mathrm{R})$ & AATTTGGCGATCCACAGCTAGTTC \\
\hline $\operatorname{Hmgr}(F)$ & CAACATCGTCACTGCCATC \\
\hline Hmgr(R) & GATGCTCAAGCTGCCTTCT \\
\hline $\operatorname{SCD} 1(\mathrm{~F})$ & TGCTGATGTGCTTCATCCTG \\
\hline SCD1(R) & GGGAAACCAGGATATTCTCC \\
\hline $\mathrm{FAS}(\mathrm{F})$ & GCCTTGCGTCACTTCCAGTTA \\
\hline FAS(R) & GCTGAATACGACCACGCACTA \\
\hline $\mathrm{ACC} \alpha(\mathrm{F})$ & TGTCCTGCCCACTTTCTTCTAT \\
\hline $\operatorname{ACC} \alpha(R)$ & TCTTGCTGTCCTCCTCTGAGTA \\
\hline $\operatorname{PPAR} \alpha(F)$ & TCAGTACATGTCTCTGTAGA \\
\hline $\operatorname{PPAR} \alpha(\mathrm{R})$ & GGTCAGGGCCCGGGTCATACTCGCGGG \\
\hline $\mathrm{HMG}-\mathrm{CoA}(\mathrm{F})$ & GGTGGTGGGACCAACCTTCT \\
\hline HMG-CoA(R) & CACGCCCCTTGAACACCTA \\
\hline CYP7A1(F) & GGTTCTTCAGGTGTGAAACT \\
\hline CYP7A1(R) & CAGAGATCTTGCCTGGCTCT \\
\hline $\mathrm{FXR}(\mathrm{F})$ & TCCGAAGAAGCATCACCAAA \\
\hline $\operatorname{FXR}(\mathrm{R})$ & CAGCCAАСАТTСССАТСТСТС \\
\hline$\beta-\operatorname{actin}(F)$ & CCACTGGCATCGTGATGGAC \\
\hline$\beta-\operatorname{actin}(\mathrm{R})$ & GCGGATGTCCACGTCACACT \\
\hline
\end{tabular}

$(P<0.05$, Table 4). After the probiotic intervention, the TC, TG, VLDL-C, LDL-C, and TBA values in the HFPB and HFPBS groups were significantly lower than those in the $\mathrm{HF}$ group $(P<0.05$, Table 4), while the TC, TG, and VLDL-C values in the HFPB group were similar to those in the $C$ group. The HFPB and HFPBS groups did not significantly differ in any of the indexes, except for TC, and the HFPBS group had a significantly higher TC value than the HFPB group $(P<0.05$, Table 4).

In the liver, the TC, TG, and TBA values in the HF group were significantly higher than those in the $\mathrm{C}$ group $(P<0.05$, Table 5). After the probiotic intervention, the values of TC, TG, and TBA in the HFPB and HFPBS group were significantly lower than those in the HF group $(P<0.05$, Table 5$)$, and the values of TG were similar to those in the $\mathrm{C}$ group. The value of TC in the HFPB group was significantly lower than that in the HFPBS group $(P<0.05$, Table 5$)$.

In the small intestine, the TC, TG, and TBA values in the $\mathrm{HF}$ group were significantly higher than those in the $\mathrm{C}$ group $(P<0.05$, Table 6). After the probiotic intervention, the values of TC, TG, and TBA in the HFPB and HFPBS groups were significantly lower than those in the $\operatorname{HF}$ group $(P<0.05$, Table 6), and the values of TC were similar to those in the $\mathrm{C}$ group $(P>0.05$, Table 6). The TC and TBA values in the HFPBS group were significantly higher than those in the HFPB group $(P<0.05$, Table 6).

\section{Histopathological Examination}

The liver in the $\mathrm{C}$ group (Figure 1A) was normal. The hepatic sinusoids were clearly visible, and the hepatic cord was regularly arranged. However, diffuse lipid changes were found in the HF group (Figure 1B). The liver cells were enlarged; the hepatic sinusoids were narrowed or even disappeared; the structure of the hepatic cord was disorganized; and some manifestations of hepatocyte edema, punctate necrosis, and infiltration of inflammatory cells were observed. The liver injury in the HFPB and HFPBS groups (Figures $\mathbf{1 C}, \mathbf{D}$, respectively) was reduced, the number of fatty liver cells was decreased, and the number of lipid droplets in the cytoplasm was also decreased sometimes to zero.

\section{Transcription Levels of Genes Associated With Lipid Metabolism in Rat Livers}

The transcription levels of the genes associated with lipid metabolism in rat livers are illustrated in Figure 2. In the HF group, the transcription of genes involved in the regulation of adipogenesis (SREBP-1C, FAS, and SCD1) and cholesterol transport and metabolism (LXR $\alpha, \mathrm{LXR} \beta, \mathrm{ABCA} 1, \mathrm{ABCG} 1$, and ABCG5) was significantly increased compared to that in the $C$ group $(P<0.05$, Figure 2$)$, while the transcription of genes involved in fatty acid $\beta$-oxidation (PPAR $\alpha$ and ChREBP) was significantly decreased.

TABLE 3 | Body weight and organ indexes.

\begin{tabular}{|c|c|c|c|c|c|c|}
\hline & Initial weight (g) & Final weight (g) & Cardiac index & Liver index & Spleen index & Renal index \\
\hline C & $141.65 \pm 11.97^{a}$ & $332.02 \pm 7.49^{a}$ & $0.32 \pm 0.02^{a}$ & $2.53 \pm 0.07^{a}$ & $0.17 \pm 0.01^{a}$ & $0.54 \pm 0.03^{a}$ \\
\hline $\mathrm{HF}$ & $142.77 \pm 16.01^{a}$ & $368.11 \pm 10.05^{b}$ & $0.33 \pm 0.04^{a}$ & $3.41 \pm 0.09^{b}$ & $0.15 \pm 0.01^{a}$ & $0.50 \pm 0.07^{a}$ \\
\hline HFPB & $138.36 \pm 15.06^{a}$ & $346.41 \pm 10.08^{c}$ & $0.30 \pm 0.03^{a}$ & $3.24 \pm 0.14^{b}$ & $0.15 \pm 0.01^{a}$ & $0.51 \pm 0.03^{a}$ \\
\hline HFPBS & $144.53 \pm 16.87^{a}$ & $362.94 \pm 9.88^{b}$ & $0.33 \pm 0.07^{a}$ & $3.27 \pm 0.15^{b}$ & $0.15 \pm 0.01^{a}$ & $0.52 \pm 0.07^{a}$ \\
\hline
\end{tabular}

Values are expressed as the mean $\pm S D(n=10)$. Different letters indicate significant differences among the groups $(P<0.05)$. 
TABLE 4 | Serum physiological and biochemical indexes.

\begin{tabular}{|c|c|c|c|c|c|c|}
\hline & $\mathrm{TC}$ & $\mathbf{T G}$ & VLDL-C & HDL-C & LDL-C & TBA \\
\hline C & $1.86 \pm 0.07^{a}$ & $1.49 \pm 0.17^{a}$ & $1.80 \pm 0.04^{a}$ & $0.65 \pm 0.5^{a}$ & $0.03 \pm 0.02^{a}$ & $31.07 \pm 0.19^{a}$ \\
\hline HF & $2.73 \pm 0.18^{b}$ & $1.95 \pm 0.10^{b}$ & $2.39 \pm 0.06^{b}$ & $0.50 \pm 0.01^{b}$ & $0.14 \pm 0.02^{b}$ & $49.43 \pm 0.17^{b}$ \\
\hline HFPB & $1.97 \pm 0.17^{a}$ & $1.53 \pm 0.21^{a}$ & $1.94 \pm 0.10^{\mathrm{ac}}$ & $0.52 \pm 0.03^{b}$ & $0.06 \pm 0.03^{a d}$ & $34.68 \pm 0.87^{c}$ \\
\hline HFPBS & $2.11 \pm 0.12^{c}$ & $1.62 \pm 0.05^{a}$ & $2.07 \pm 0.11^{\mathrm{c}}$ & $0.51 \pm 0.03^{b}$ & $0.09 \pm 0.04^{d}$ & $38.13 \pm 0.33^{c}$ \\
\hline
\end{tabular}

Values are expressed as the mean $\pm S D(n=10)$. Different letters indicate significant differences among the groups $(P<0.05)$.

TABLE 5 | Liver physiological and biochemical indexes.

\begin{tabular}{lccc}
\hline & TC & TG & TBA \\
\hline $\mathrm{C}$ & $0.025 \pm 0.004^{\mathrm{a}}$ & $0.098 \pm 0.016^{\mathrm{a}}$ & $1.556 \pm 0.024^{\mathrm{a}}$ \\
$\mathrm{HF}$ & $0.065 \pm 0.007^{\mathrm{b}}$ & $0.134 \pm 0.017^{\mathrm{b}}$ & $1.740 \pm 0.017^{\mathrm{b}}$ \\
$\mathrm{HFPB}$ & $0.043 \pm 0.002^{\mathrm{c}}$ & $0.100 \pm 0.007^{\mathrm{a}}$ & $2.020 \pm 0.020^{\mathrm{c}}$ \\
HFPBS & $0.043 \pm 0.007^{\mathrm{c}}$ & $0.090 \pm 0.013^{\mathrm{a}}$ & $1.966 \pm 0.024^{\mathrm{c}}$
\end{tabular}

Values are expressed as the mean $\pm S D(n=10)$. Different letters indicate significant differences among the groups $(P<0.05)$.

TABLE 6 | Small intestine physiological and biochemical indexes.

\begin{tabular}{lccc}
\hline & TC & TG & TBA \\
\hline $\mathrm{C}$ & $0.051 \pm 0.009^{\mathrm{a}}$ & $0.181 \pm 0.072^{\mathrm{a}}$ & $1.270 \pm 0.266^{\mathrm{a}}$ \\
$\mathrm{HF}$ & $0.077 \pm 0.019^{\mathrm{b}}$ & $0.308 \pm 0.034^{\mathrm{b}}$ & $1.480 \pm 0.183^{\mathrm{b}}$ \\
HFPB & $0.052 \pm 0.010^{\mathrm{a}}$ & $0.237 \pm 0.011^{\mathrm{c}}$ & $1.690 \pm 0.262^{\mathrm{c}}$ \\
HFPBS & $0.059 \pm 0.006^{\mathrm{a}}$ & $0.272 \pm 0.013^{\mathrm{d}}$ & $1.790 \pm 0.259^{\mathrm{d}}$ \\
\hline
\end{tabular}

Values are expressed as the mean $\pm S D(n=10)$. Different letters indicate significant differences among the groups $(P<0.05)$.

Upregulated LXR $\alpha, \mathrm{LXR} \beta, \mathrm{ABCA} 1, \mathrm{ABCG} 1, \mathrm{SCD} 1$, and $\operatorname{PPAR} \alpha$ gene transcription levels and downregulated SREBP-1C and FAS gene transcription levels were observed in the HFPB and HFPBS groups relative to those in the HF groups $(P<0.05$, Figure 2). Furthermore, the ABCG5, Hmgr, and HMG-CoA gene transcription in the HFPB group were significantly higher than that in the HF group $(P<0.05$, Figure 2$)$.

However, in the HFPB group, the transcription of genes involved in the regulation of cholesterol transport and metabolism (LXR $\alpha$, LXR $\beta$, ABCA1, ABCG1, ABCG5, Hmgr, and $\mathrm{HMG}-\mathrm{CoA}$ ) was significantly increased compared to that in the HFPBS group $(P<0.05$, Figure 2$)$. Furthermore, the SREBP-1C and PPAR $\alpha$ gene transcription levels in the HFPB group were significantly higher than that in the HFPBS group $(P<0.05$, Figure 2$)$.

\section{Transcription Levels of Genes Associated With Lipid Metabolism in the Small Intestine of Rats}

The transcription levels of the genes associated with lipid metabolism in the small intestine of rats are illustrated in Figure 3. Compared to the $\mathrm{C}$ group, feeding the rats a high-fat diet significantly increased the transcription of genes involved in cholesterol transport and metabolism $(\mathrm{LXR} \alpha, \mathrm{LXR} \beta, \mathrm{ABCA} 1$, and ABCA5) $(P<0.05$, Figure 3).
In the HFPB group, the transcription of genes involved in the regulation of adipogenesis (SCD1 and PPAR $\alpha$ ) and cholesterol transport, and the metabolism $(\mathrm{LXR} \alpha, \mathrm{LXR} \beta, \mathrm{ABCA} 1$, and ABCA5) was significantly increased compared with that in the HF group $(P<0.05$, Figure 3).

In the HFPBS group, the transcription of genes involved in cholesterol transport and metabolism (ABCA1) was significantly increased, and the transcription of LXR $\beta, A B C A 5, F X R$, and CYP7A1 was decreased compared with that in the HF group $(P<0.05$, Figure 3). Furthermore, transcription in the HFPBS group was significantly lower than that in the HFPB group $(P<0.05$, Figure 3).

\section{DISCUSSION}

Based on previous studies, this study attempts to analyze the multifaceted mechanism by which probiotics reduce lipid levels in their hosts by evaluating the transcription of the genes involved in the LXR, AMPK, and FXR axes of lipid metabolism. In addition, the effects of single and combined probiotic-fermented dairy products on the lipid level and transcription of lipid metabolism-related genes in hyperlipidemic rats were compared. The results show that probiotic-fermented dairy products significantly reduced the levels of TC and TG in the serum, liver, and small intestine of the rats and reduced lipid accumulation in the liver. There were no significant differences between the single and combined probiotic-fermented dairy products. Other reports have shown that L. acidophilus, L. plantarum 9-41-A, L. fermentum M1-16, and L. plantarum MA2 are able to reduce the liver TC and lipid levels (Fukushima and Nakano, 1996; Wang et al., 2009; Xie et al., 2011), while another study also showed that the probiotic L. gasseri SBT2055 can reduce not only abdominal visceral fat but also subcutaneous fat (Kadooka et al., 2010).

Compared to the HF group, the transcription of genes involved in the LXR axis in the liver and small intestine was significantly upregulated in both the HFPB and HFPBS groups. LXRs are the main "receptors" maintaining the relative stability of intracellular cholesterol. The LXRs predominantly maintain cholesterol balance through the following processes: (1) inhibition of the intestinal absorption of cholesterol in food; (2) regulation of the synthesis and absorption of cholesterol in the liver; (3) regulation of the reverse transport of cholesterol; and (4) promotion of the transformation of cholesterol to bile acid and cholesterol excretion through bile (Trasino et al., 2013). The transcription of $\mathrm{LXR} \alpha, \mathrm{LXR} \beta, \mathrm{ABCA} 1, \mathrm{ABCG} 1$, and ABCG5 in the liver and small intestine in the HFPB and 

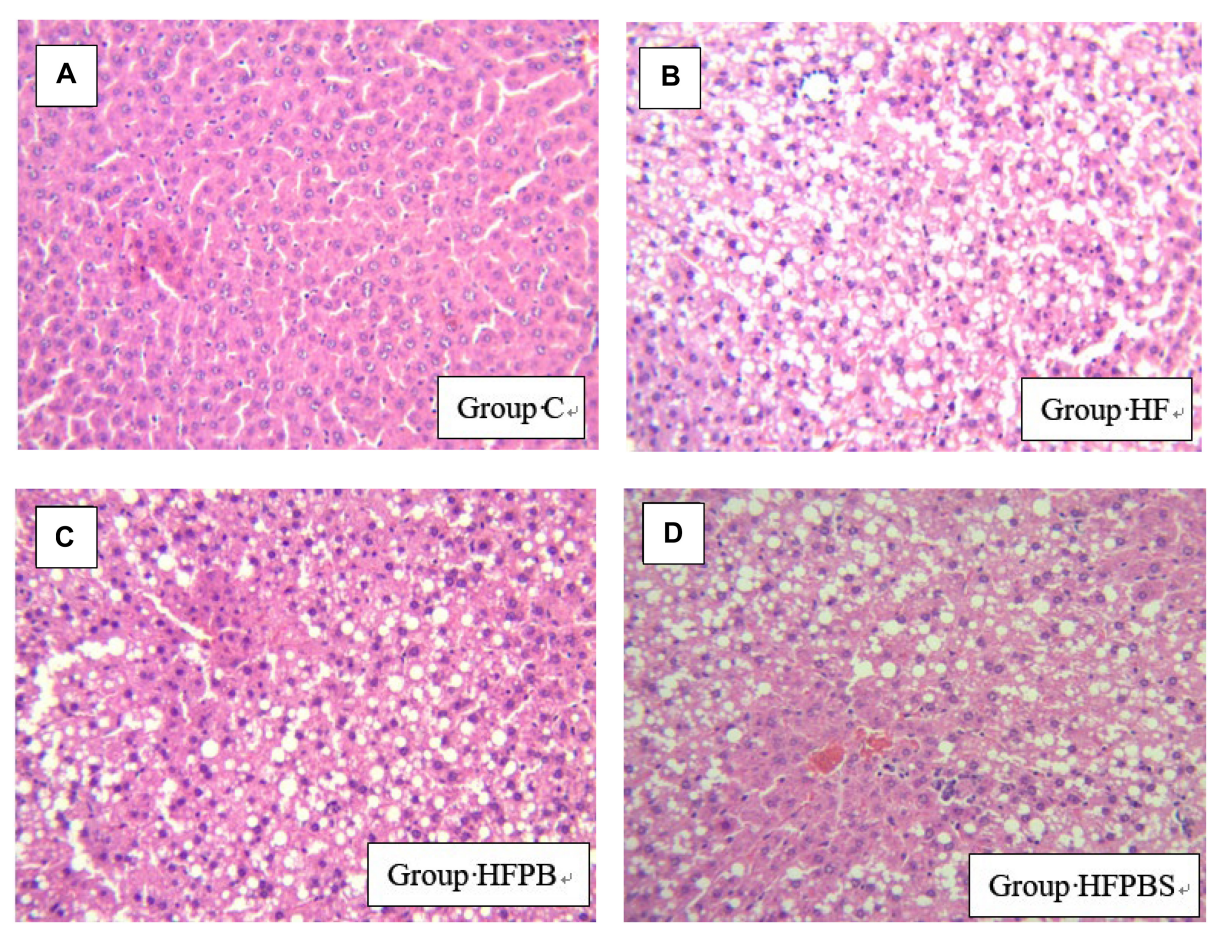

FIGURE 1 | Histopathological examination of liver tissue showing damage and arrangement of hepatocytes and fat vacuoles (100 $\times$ magnification) ( $n=10)$. (A) Tissue from the $\mathrm{C}$ group showing a normal liver with no lipid deposits. (B) Liver tissue from the HF group showing the presence of steatosis. (C) Tissue from the HFPB group showing the presence of steatosis. (D) Cells from the HFPBS group containing many large fat vacuoles.

HFPBS group was significantly higher than that in the $\mathrm{HF}$ group $(P<0.05)$. The transcription of the LXR genes in the liver and small intestine of the rats was considered activated by the probiotics, thus upregulating the transcription of genes involved in the LXR axis. The upregulated transcription of ABCA1 and ABCG1 increased cellular cholesterol efflux to HDL$\mathrm{C}$ in the serum, which increased RCT. The upregulation of ABCG5 promotes the secretion and excretion of cholesterol in the hepatobiliary tube. Furthermore, upon activation, the LXR in the small intestine has been observed to be activated, and the transcription of NPC1 L1 was downregulated (Wang et al., 2006), which reduced the absorption of cholesterol, increased the transcription of ABCG5, and mediated the flow of sterols in intestinal cells to the absorption of cholesterol in the intestinal cavity (Wang et al., 2006). Therefore, the content of TC in the serum and liver in the HFPB and HFPBS groups (Table 4) was significantly lower than that in the HF group $(P<0.05)$, while the content of TBA was significantly increased $(P<0.05)$. In addition, in the intestinal tract, the upregulation of the FXR and CYP7A1 genes as a result of the administration of the probiotics-fermented milks was not significantly compared with that in the HF group, and that in the HFPBS group was significantly lower than that in the HF group. Thus, it is possible that a decrease in the host TC caused by probiotics may not be achieved by affecting the genes involved in the FXR axis.

Regarding the transcription of triglyceride-related genes in the liver and small intestine, there were no significant differences in AMPK transcription between the HFPB and HFPBS groups compared with the HF group, but the transcription of $\operatorname{LXR} \alpha$, PPAR $\alpha$, and SCD-1 in the liver was significantly upregulated, while the transcription of ChREBP and FAS was significantly downregulated $(P<0.05)$. First, ChREBP is a target gene of LXRs and can upregulate the transcription of liver-type pyruvate kinase. ChREBP and liver-type pyruvate kinase play important roles in the conversion of carbohydrates to lipids. A study by Uyeda et al. (2002) showed that LXR agonists can promote the conversion of carbohydrates to fat in the liver, leading to the accumulation of fat in this organ. However, in the current study, the probiotics activated the transcription of the LXR gene but reduced the transcription of the ChREBP gene, which inhibited the transformation of carbohydrates into fat in the liver and reduced hepatic fat accumulation. Similarly, the treatment with the probiotics resulted in an upregulation of the SCD-1 gene in the liver. SCD-1 reduces free saturated fatty acids in liver cells, thus reducing the induction of lipid peroxidation in liver cells. Second, the treatment with the probiotics resulted in the upregulation of the LXR $\alpha$ and PPAR $\alpha$ genes in the liver, and a study by Trasino et al. (2013) showed that LXR $\alpha$ can inhibit the differentiation of mesenchymal stem cells into adipocytes through $\mathrm{Wnt} / \beta$-catenin ( $\beta$-catenin) signaling. PPAR $\alpha$ regulates peroxidase, fatty acid transport, and mitochondrial fatty acid $\beta$-oxidation metabolism gene transcription and expression, thereby promoting the decomposition, absorption, and utilization of fatty acids (Zhou et al., 2008). Therefore, the treatment with the probiotics did not directly activate AMPK, 

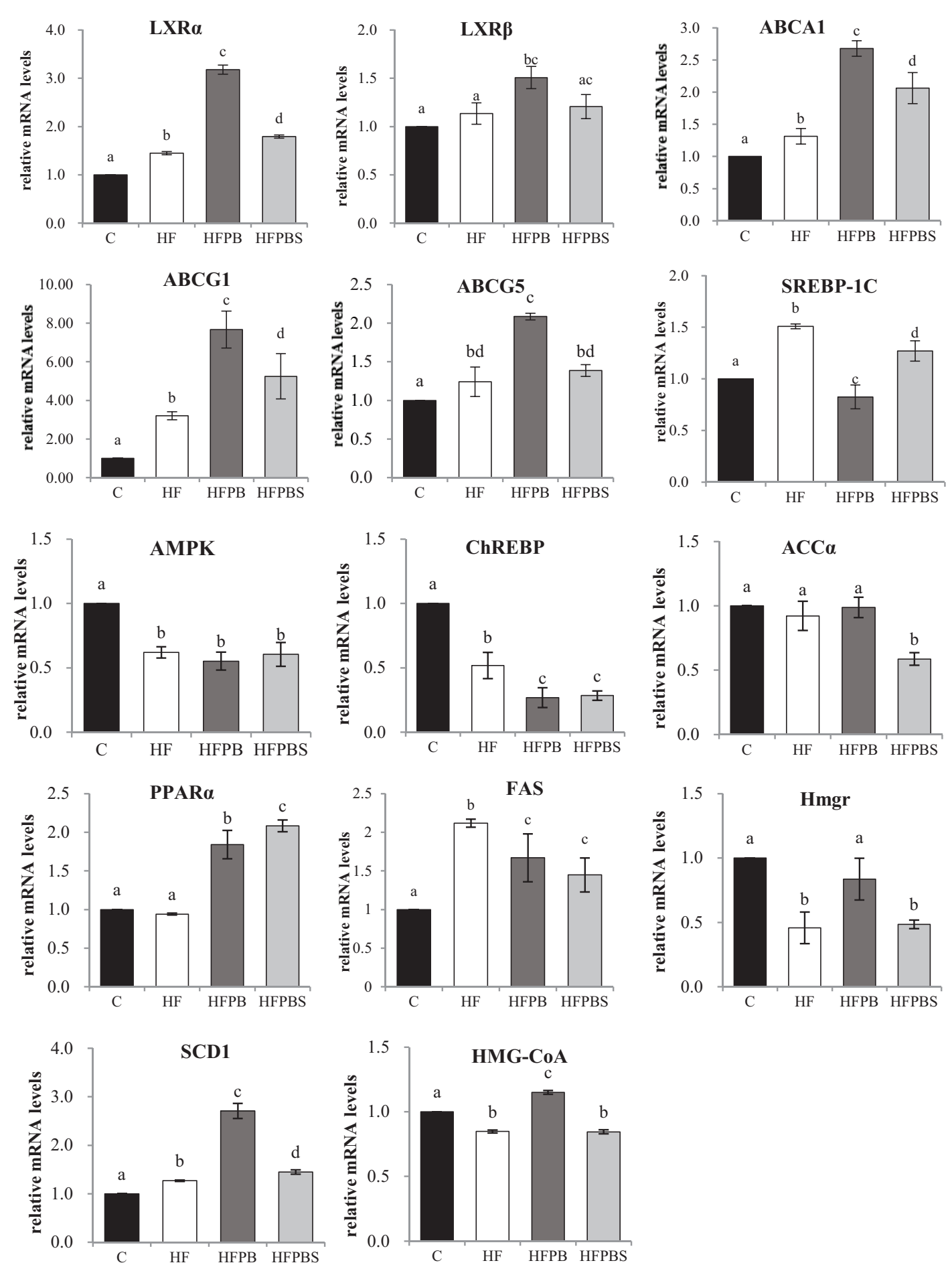

FIGURE 2 | Effects of diet on the transcription of genes related to lipid metabolism in freshly isolated livers. The total RNA was extracted, reverse transcribed to cDNA, and analyzed to detect the transcription of genes using RT-PCR. Gene transcription is reported as the mean \pm SD $(n=6)$ of the fold change relative to the $\mathrm{C}$ group. Error bars with different letters indicate significant differences $(P<0.05)$.

but the level of TG in the rat serum, liver, and small intestine was significantly reduced by the activation of the transcription of PPAR $\alpha$ and $\operatorname{LXR} \alpha(P<0.05)$.
Notably, the effects of the probiotic intake showed the same trend in both the rat liver and small intestine in the rats fed the HF diet only compared to the rats in the $\mathrm{C}$ group (normal 

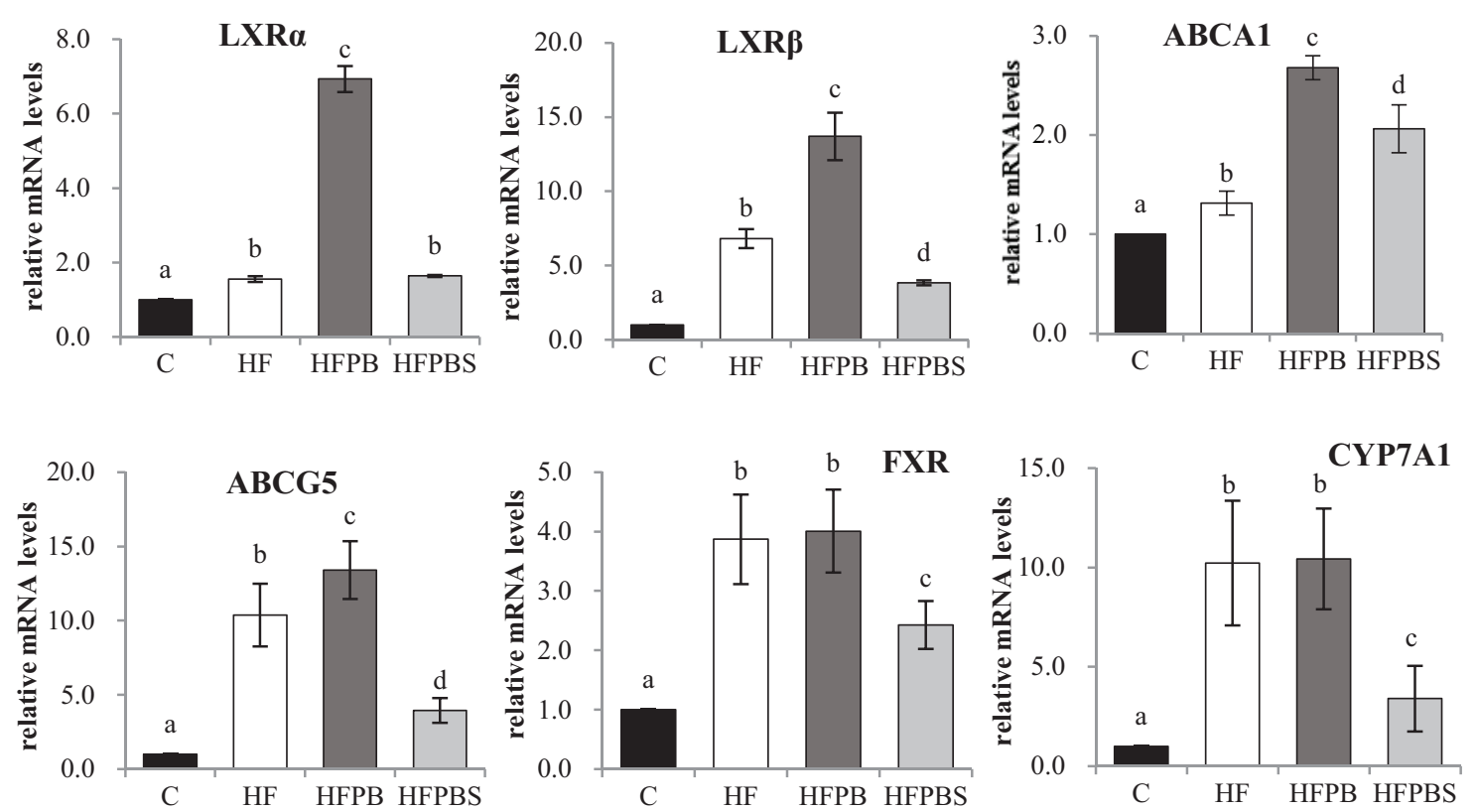

FIGURE 3 | Effects of diet on the transcription of genes related to lipid metabolism in the small intestine. The total RNA was extracted from freshly isolated small intestines, reverse transcribed to cDNA, and analyzed to detect the transcription of genes using RT-PCR. Gene transcription is reported as the mean \pm SD ( $n=6)$ of the fold change relative to the $\mathrm{C}$ group. Error bars with different letters indicate significant differences $(P<0.05)$.

diet), i.e., increased transcription of most genes. The possible reasons for these results are as follows. First, the daily diet was an important factor affecting lipid metabolism. When a large amount of fat is provided in the diet, the transcription levels of the genes maintaining homeostasis in lipid metabolism (e.g., LXRs, AMPK, and FXR) were also upregulated to maintain the lipid levels. The HF, HFPB, and HFPBS groups were fed a high-fat diet; thus, these groups showed similar trends. However, after the probiotics administration intervention, the lipid metabolism disorder induced by the high-fat diet was alleviated, further indicating that the administration of the probiotics was effective. Second, the degree of hepatocytes steatosis that the histopathological examination showed was not sufficient to stop lipid metabolism, so rats could still undergo feedback regulation when they ingested a large quantity of lipids. In addition, compared with the HF group, hepatocytes steatosis was obviously alleviated in the HFPB and HFPBS group, further suggesting that the damage was reversible and that the probiotics administration intervention was effective.

Furthermore, there were no significant differences in the physiological and biochemical indexes between the HFPB and HFPBS groups, except TC level, and the ability of the rats in the HFPB group to regulate the transcription of cholesterol metabolism-related genes was significantly better than that in those in the HFPBS group $(P>0.05)$. Notably, LXR agonists can cause the accumulation of liver fat and an increase in TG levels in the plasma, which mainly occurs because LXRs induce the transcription of SREBP1c. SREBP1c is a major transcription factor of the fat synthesis gene, which activates a variety of enzymes involved in fatty acid and triglyceride synthesis. Therefore, researchers believe that new LXR agonists can regulate some repressor and auxiliary activators to selectively upregulate ABCA1 without upregulating the transcription of SREBP1c (Beyea et al., 2007). The transcription of SREBP1c was significantly downregulated in the HFPB group but significantly upregulated in the HFPBS group. Therefore, we believe that the long-term use of L. rhamnosus LV108-fermented dairy products has a better effect on reducing lipid accumulation in the liver, small intestine, and blood.

\section{CONCLUSION}

The milk fermented with a single probiotic (L. rhamnosus LV108) and the combined probiotic (L. rhamnosus LV108, L. casei grx12, and $L$. fermentum grx08)-fermented milk significantly improved the lipid levels and visceral lipid accumulation in hyperlipidemic rats. At the physiological and biochemical levels, there were no significant differences between the single probiotic- and combined probiotic-fermented milks in the hyperlipidemic rats; however, the regulation of the transcription of lipid metabolismrelated genes caused by the single probiotic-fermented milk was more effective than that by the combined probioticfermented milk.

\section{ETHICS STATEMENT}

This study was carried out in accordance with the recommendations of the China Institutional and National Guidelines, Ethics Committee of the Yangzhou University. 
The protocol was approved by the Ethics Committee of the Yangzhou University.

\section{AUTHOR CONTRIBUTIONS}

YW, BY, and RG participated in the design of this study. YW and BY contributed equally to this work and performed the statistical analysis. YoH, WX, YiH, CW, and FG carried out the study and

\section{REFERENCES}

Beyea, M. M., Heslop, C. L., Sawyez, C. G., Edwards, J. Y., Markle, J. G., Hegele, R. A., et al. (2007). Selective up-regulation of LXR-regulated genes ABCA1, ABCG1, and APOE in macrophages through increased endogenous synthesis of 24(S),25-epoxycholesterol. J. Biol. Chem. 282, 5207-5216. doi: 10.1074/jbc. M611063200

Chen, D. W. (2014). Screening of Auxiliary Hypolipidemic Properties of Probiotic and its Impact on the Intestinal Microbiota of Hyperlipidemia Rat Model. Dissertation, Yangzhou University, Yangzhou, CHN.

Fukushima, M., and Nakano, M. (1996). Effects of a mixture of organisms, Lactobacillus acidophilus or Streptococcus faecalis on cholesterol metabolism in rats fed on a fat- and cholesterol-enriched diet. Br. J. Nutr. 76, 857-867. doi: 10.1079/bjn19960092

Geyeregger, R., Zeyda, M., and Stulnig, T. M. (2006). Liver X receptors in cardiovascular and metabolic disease. Cell Mol. Life Sci. 63, 524-539. doi: 10. 1007/s00018-005-5398-3

He, M., and Shi, B. (2017). Gut microbiota as a potential target of metabolic syndrome: the role of probiotics and prebiotics. Cell Biosci. 7:54. doi: 10.1186/ s13578-017-0183-1

Huang, Y.-J., Liang, W.-X., Liu, D., Chen, D.-W., Lu, J., and Gu, R.-X. (2013). Screening of cholesterol- reducing probiotic isolated from the intestine of the longevity crowd in vitro. China Dairy Ind. 41, 13-16.

Huang, Z., Mu, C., Chen, Y., Zhu, Z., Chen, C., Lan, L., et al. (2015). Effects of dietary probiotic supplementation on LXRalpha and CYP7alphal gene expression, liver enzyme activities and fat metabolism in ducks. Br. Poult. Sci. 56, 218-224. doi: 10.1080/00071668.2014.1000821

Kadooka, Y., Sato, M., Imaizumi, K., Ogawa, A., Ikuyama, K., Akai, Y., et al. (2010). Regulation of abdominal adiposity by probiotics (Lactobacillus gasseri SBT2055) in adults with obese tendencies in a randomized controlled trial. Eur. J. Clin. Nutr. 64, 636-643. doi: 10.1038/ejcn.2010.19

Liu, D.-F., Xue, M., Sang, J., Chen, D.-W., He, Y., Li, Z.-H., et al. (2016). Screening of lactic acid bacteria with high adhesion and its effect on the activity of immune cells. China J. Microecol. 28, 869-874.

Mann, G. V. (1974). Studies of a surfactant and cholesteremia in the Maasai. Am. J. Clin. Nutr. 27, 464-469. doi: 10.1093/ajcn/27.5.464

Patel, J. J., Butters, O. R., and Arnett, T. R. (2014). PPAR agonists stimulate adipogenesis at the expense of osteoblast differentiation while inhibiting osteoclast formation and activity. Cell Biochem. Funct. 32, 368-377. doi: 10. $1002 /$ cbf.3025

Song, C., Kokontis, J. M., Hiipakka, R. A., and Liao, S. (1994). Ubiquitous receptor: a receptor that modulates gene activation by retinoic acid and thyroid hormone receptors. Proc. Natl. Acad. Sci. U.S.A. 91, 10809-10813. doi: 10.1073/pnas.91. 23.10809

Tall, A. R., Yvan-Charvet, L., Terasaka, N., Pagler, T., and Wang, N. (2008). HDL, $\mathrm{ABC}$ transporters, and cholesterol efflux: implications for the treatment of atherosclerosis. Cell Metab. 7, 365-375. doi: 10.1016/j.cmet.2008.03.001

Trasino, S. E., Dawson, H. D., Urban, J. F. Jr., Wang, T. T., and Solano-Aguilar, G. (2013). Feeding probiotic Lactobacillus paracasei to Ossabaw pigs on a high-fat diet prevents cholesteryl-ester accumulation and LPS modulation of the Liver X receptor and inflammatory axis in alveolar macrophages. J. Nutr. Biochem. 24, 1931-1939. doi: 10.1016/j.jnutbio.2013.06.001 collected important background information. YW drafted the manuscript. All authors read and approved the final manuscript.

\section{FUNDING}

This work was supported by the Strategic Priority Research Program of the National Natural Science Foundation of China (31571855).

Tu, H., Okamoto, A. Y., and Shan, B. (2000). FXR, a bile acid receptor and biological sensor. Trends Cardiovasc. Med. 10, 30-35. doi: 10.1016/s10501738(00)00043-8

Uyeda, K., Yamashita, H., and Kawaguchi, T. (2002). Carbohydrate responsive element-binding protein (ChREBP): a key regulator of glucose metabolism and fat storage. Biochem. Pharmacol. 63, 2075-2080. doi: 10.1016/s0006-2952(02) 01012-2

Wang, N., Ranalletta, M., Matsuura, F., Peng, F., and Tall, A. R. (2006). LXRinduced redistribution of ABCG1 to plasma membrane in macrophages enhances cholesterol mass efflux to HDL. Arterioscler. Thromb. Vasc. Biol. 26, 1310-1316. doi: 10.1161/01.atv.0000218998.75963.02

Wang, Y., Xu, N., Xi, A., Ahmed, Z., Zhang, B., and Bai, X. (2009). Effects of Lactobacillus plantarum MA2 isolated from tibet kefir on lipid metabolism and intestinal microflora of rats fed on high-cholesterol diet. Appl. Microbiol. Biotechnol. 84, 341-347. doi: 10.1007/s00253-009-2012-x

Willy, P. J., Umesono, K., Ong, E. S., Evans, R. M., Heyman, R. A., and Mangelsdorf, D. J. (1995). LXR, a nuclear receptor that defines a distinct retinoid response pathway. Genes Dev. 9, 1033-1045. doi: 10.1101/gad.9. 9.1033

Xie, N., Cui, Y., Yin, Y.-N., Zhao, X., Yang, J.-W., Wang, Z.-G., et al. (2011). Effects of two Lactobacillus strains on lipid metabolism and intestinal microflora in rats fed a high-cholesterol diet. BMC Compl. Altern. Med. 11:53. doi: 10.1186/14726882-11-53

Yao, F., Zhang, M., and Chen, L. (2016). 5'-Monophosphate-activated protein kinase (AMPK) improves autophagic activity in diabetes and diabetic complications. Acta Pharm. Sin. B 6, 20-25. doi: 10.1016/j.apsb.2015.07.009

Yoshikawa, T., Shimano, H., Amemiya-Kudo, M., Yahagi, N., Hasty, A. H., Matsuzaka, T., et al. (2001). Identification of liver X receptor-retinoid X receptor as an activator of the sterol regulatory element-binding protein $1 \mathrm{c}$ gene promoter. Mol. Cell. Biol. 21, 2991-3000. doi: 10.1128/MCB.21.9.2991-3000. 2001

Zelcer, N., and Tontonoz, P. (2006). Liver X receptors as integrators of metabolic and inflammatory signaling. J. Clin. Invest. 116, 607-614. doi: 10.1172/ JCI27883

Zhang, Y., Du, R.-T., He, Q.-W., Li, H., and Zhang, H.-P. (2012). Effect of Lactobacillus casei Zhang administration on liver lipids metabolism of high-Fat diet induced hypercholesterolemia rats. Sci. Agric. Sin. 45, 943-950.

Zhou, J., Febbraio, M., Wada, T., Zhai, Y., Kuruba, R., He, J., et al. (2008). Hepatic fatty acid transporter Cd36 is a common target of LXR, PXR, and PPAR gamma in promoting steatosis. Gastroenterology 134, 556-567. doi: 10.1053/j.gastro. 2007.11.037

Conflict of Interest Statement: The authors declare that the research was conducted in the absence of any commercial or financial relationships that could be construed as a potential conflict of interest.

Copyright (C) 2019 Wa, Yin, He, Xi, Huang, Wang, Guo and Gu. This is an open-access article distributed under the terms of the Creative Commons Attribution License (CC BY). The use, distribution or reproduction in other forums is permitted, provided the original author(s) and the copyright owner(s) are credited and that the original publication in this journal is cited, in accordance with accepted academic practice. No use, distribution or reproduction is permitted which does not comply with these terms. 